\title{
Glove Sensor with AI system for Deaf and Blind People to Ease of Communication
}

\author{
T.Suresh, P. Gunasekhar, Z. Brijet, Jasti Tejas
}

\begin{abstract}
Deaf and blind people find very difficult to communicate to other people. The latest technological development helps the people having disabilities particularly deaf and blind people to communicate to other people. These people can able to communicate with the help of their sense of touch. So the advance sensors integrating with the recent technology assists the people with disability for communicating with the other people. In this paper Glove based sensors combined with the Artificial Intelligence is demonstrated so that the deaf and blind people can communicate to other people in a big conference or any public places. It is also proved that the device developed is a low cost wearable device which is affordable to such a weaker section of people having disabilities. Raspberry-pi and mobile device are cantered around the sensors to process and communicate. With the help of the device demonstrated in this paper people with disabilities specially deaf and blind can involve freely in day to day regular activities.
\end{abstract}

Keywords: Index Terms-wearable devices, Glove with sensors. Artificial intelligence, deaf and blind people,

\section{INTRODUCTION}

The information can be passed from one person to another through communication and one person can understand the other person via communication. It is nothing but the way of transmitting and sharing ideas, opinions, thoughts, views, suggestions etc. from one person to another or one firm to another, as described in [1]. But deaf and dump people cannot communicate among them or other people without disabilities, but they can communicate through the other form of communication such as gestures. These aspects are:

1) A sender, who sends the message.

2) A message, that is the content of the communication.

3) A recipient, who receives the message.

Revised Manuscript Received on November 17, 2019

* Correspondence Author

Dr.T.Suresh, Professor \& Head, Department of Electronics and Communication Engineering, R.M.K Engineering college, Thiruvallur district, Tamilnadu, India. Email: hod.ece@ rmkec.ac.in

P. Gunasekhar, Assistant Professor, Department of Electronics and Communication Engineering, R.M.K Engineering college, Thiruvallur district, Tamilnadu, India.

Email: pgr.ece@rmkec.ac.in

Z. Brijet, Assistant Professor, Department of EIE, Velamma Engineering College, Thiruvallur district, Tamilnadu, India. Email fiojet@yahoo.com

Jasti Tejas, Programmer Analysts, Cognizant Technology Solutions Chennai, India. Email: tejasjasti@gmail.com Accessibility.

4) A context, that is the general situation and the particular circumstances in which every communication happens.

5) A common code to sender and recipient.

6) A communication channel (physical or

psychological) between sender and recipient.

Communication has a key role in our daily life and in particular in daily activities of people with disabilities, since it represents a means of inclusion and of integration in the society and a way to enhance their independence [2]. It takes on further importance in severe disability cases like deaf and blindness that is the combination of blindness and deafness. Deaf-blind people's communication is very complex, because they use other form of communication to make their expression understandable so that they can keep in touch with other people, avoiding isolation from other people. For deaf and blind people to interact with other people, caretaker is needed to interpreter their language with the other people in the world [3].

Communication is also one of the activities which are considered as crucial issues at the basis of daily activities of deaf-blind people and of people with disabilities in general, since they are essential in order to have a social life [4]. The other most important activities that have to be considered to improve the quality of life of people with disabilities are the following ones:

- Learning: central hub and base for communication [5], especially for people who are deaf-blind since birth.

- Moving: essential thing regarding daily living of both independence and finishing activities, in both indoor [6] and outdoor, and in urban and extra-urban circumstances [7].

Despite the severe pathology, there are different methods of interaction for deaf-blind people. Many of them are not verbal and can be alternatively applied depending on the total or partial loss of sight and hearing. In general case, most of the systems mainly use the touch sense to communicate among people. These codes are based on various complex language, which is based on residual capacities or age possessed [8], while, in the other case, vibrations and tactile feedbacks can be combined with some visual cues [9]. Most often it is not possible to use a real language code In these cases, there are a lot of communication forms based,

for example, on gesture or body movements [10], even in rehabilitation context [11].

Today, the perspectives are changed thanks to the introduction of modern assistive technologies that are products,

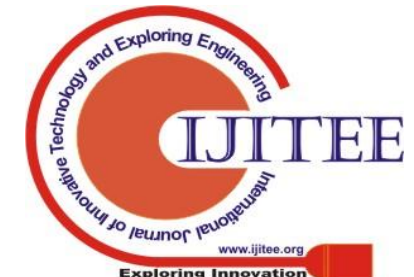


devices or systems, which allow overcoming existing device barriers. This is the context where the Glove sensor project was born. Glove-sensor developed in this paper is an affordable very low-cost device supporting the users to independently interact with the other people in the world, make them directly communicate with other people, without the need of any help of another people who acts as an interpreter [12].

Despite moving is an important issue, social communication is a strong and marked desire. This is why there is the need to extend the approach proposed in the state-of-the-art projects and move the context in

a natively distributed environment to handle one-to-many and many-to-many communications.

For a need due to the obvious difficulties of moving of the users in question, the requirement of extending the project in a distributed environment spontaneously arises. In this paper, attention is given to extend the Glove-sensor's architecture [13] with the aim of enabling communication between many users exploiting the potentiality of tuple centers as coordination media.

The rest of this paper is structured as follows. Section II briefly describes the background, by presenting some related work. Section III illustrates a scenario in which a many-to-many communication is required and Section IV deals with the architecture of the proposed system. Section $\mathrm{V}$ describes the Architecture with Tuple Center and, finally, Section VI concludes the paper with some final remarks and future work.

\section{BACKGROUND AND RELATED WORK}

In this section, we briefly introduce some work related to our case study, presenting some cases based on different communication devices for deaf-blind people. Maybe the most famous methods of communication for blind people is Braille, a tactile reading/writing system used by people who are blind or visually impaired, developed by Louis Braille, in the first half of the XIX century. During the years, different studies concern the development of Braille devices. Sarkar et al. The device developed in [14] called SPARSHA, which is a very useful low cost Braille device for blind and deaf people. This device allows interaction with the other blind and deaf people. In [15] the authors presented a device called MyVox, which is a device consists of a portable keyboard, a speaker and a Braille refreshable for the communication between two deaf and blind people, based on the ARM-based computer system.

Various analysis have been exploited the feasibility of the use of wearable devices, especially gloves, to give input and output through the gloves which enables suitable interaction among deaf and blind people. These devices are based on different approaches, depending on the communication methods.

A first approach exploits the potentiality of using Braille system on wearable devices. Choudhary et al. in

[16] explained the benefit of a smart glove to convert the text into Braille alphabet (and vice-versa), and to communicate the message via shot message system (SMS) of mobile device in order to establish a remote contact. A user can convey messages through capacitive touch sensors and she or he can interpret and perceive incoming messages by feedback patterns of mini vibration motors on the glove's dorsal side.

[17] A second one consists of using dactylology, which represents the letters in the normal writing system, and also numeral systems, using only the hands. Every spoken language has its corresponding finger spelling so proposed technical solutions will vary depends on the detail of the different alphabets. A first example is explained in [17], a robotic handmade of aluminium. This robotic prosthesis is projected vertically. Fingers, independent of each other, have been built to carry out human-like movements. There is a microprocessor to control the hand in the box underneath the device.

[19] Another approach takes advantage of the sign language, which conveys communication, as opposed to spoken language. This communication involves combining hand shapes; movement and orientation of the arms, hands and body, and expression of face all together and finally express a speaker's thoughts. Like dactylology,

[21] the sign language varies depending on the spoken language. Thomas Pryor and Navid Azodi have developed a device which consists of a pair of gloves, named 'SignAloud'. This device can translate the gestures of sign language into audible spoken English. Each glove in this device consists of sensors for recording hand position and movement and makes use of Bluetooth technology to send data to a centralized computing system which matches the gesture with the word or phrase associated. Finally, the computer vocalizes the word or phrase aloud, thanks to a text-to-speech system. Instead, Brashear et al. [18] propose a Sign Language recognition system that combines a first person camera view put, for example, in the brim of a hat, and accelerometer that are in the gloves. This combination allows collecting complementary and slightly overlapping information, helping to disambiguate noisy data in order to improve recognition accuracy.

[22] Further approaches are based on deaf and blind manual alphabets. During the years different alphabets have been proposed, each one of them thought to be helpful in different situation. One of the most famous one is the Lorm Alphabet, in which every letter of the Roman alphabet is associated with a movement of the palm of the hand. In a common case, the deaf-blind person shows (usually the right one ) the palm to the speaker, the speaker points into the hand with the tips of his finger. Gollner et al. in [19] proposed a system in which a glove can translate the hand-touch alphabet into text. The pressure sensors act as the input device of the glove while the output can be derived from the matrix of vibrating motors. Another example is the Malossi method, designed 
by Eugenio Malossi, himself affectionate by deafness. This method requires the knowledge of the written Italian language. Each phalanx of the hand is associated with a letter of the alphabet, becoming a kind of typewriter. Thus, the word is composed by touching or pinching the phalanges or the palm. This method is generally used by people who have learned reading and writing before becoming deaf-blind. DbGlove is a system that supports deaf-blind people in order to make them interact with the others through the Malossi alphabet but it also supports other alphabets such as Braille. The interaction is managed by the software that translates text into tactile signals and vice-versa. Multi-modal feedback can also be provided by this system for various standard output devices to support interaction between not only deaf and blind people but also people without disabilities. In this context, in this paper a glove sensor as shown in Figure 1, is proposed, which is an affordable wearable device, operates as an assistive technology for deaf and blind people. This device is a low cost fully autonomous device, which can support deaf and blind people to communicate with other people in the world, ensures them directly interact with other people, without any assistant of other people who acts as an interpreter. This system consists of glove with in-built MPR121 capacitive touch sensors connected with Raspberry Pi through $\mathrm{I}^{2} \mathrm{C}$ interface, and communicated to mobile device for developing Android application.

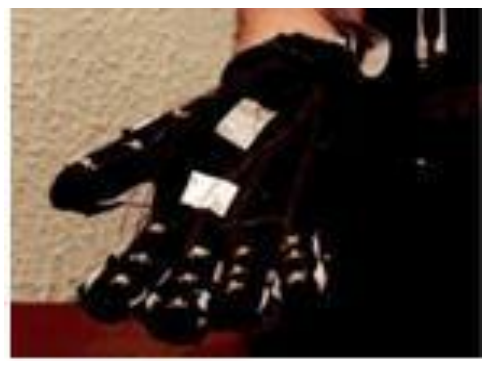

Figure 1. Glove Sensor

\section{SCENARIO} communication many-to-many is required and we discuss some issues related to this case. In this case, a typical example of many-to-many communication will be taken into account, which is a conference for deaf-blind people. This type of conference has a real consideration as conferences between/for deaf-blind people really take place even if not massively. They are handled differently based on the type and the severity of the disability. Some of them use Braille bars while others have operators that follow groups of people. Instead, in conference for people with severe disability, for example deaf-blindness, people are assisted and followed individually by an operator.

In Figure 2 there is a client-server architecture which sums up a possible infrastructure that allows communication and coordination between many deaf-blind people.
In this section we present a scenario in which a

We also want to highlight the problem of identity in distributed context. Considering the following concepts:

- Number of people around the user.

- Position of people and where attention is drawn at a given moment.

- Identity of people in front of the user.

- Facial expressions and non-verbal sign.

- Understand whether the person in front is known.

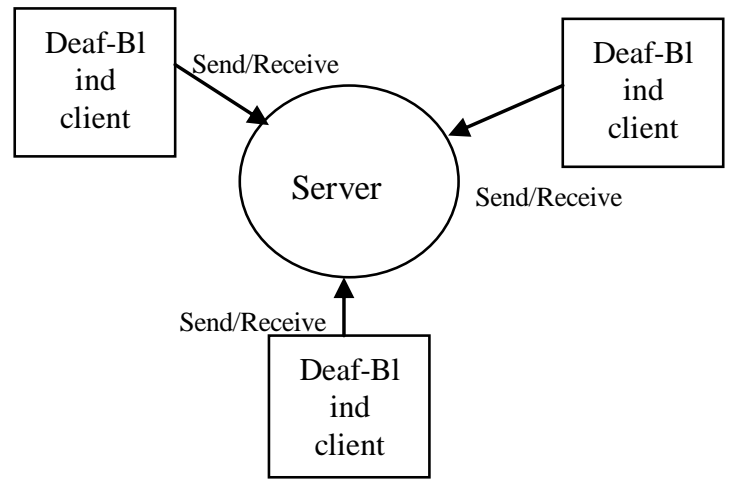

Fig. 2. Our scenario

These concepts are easy for people with no disabilities, but they are hardly acquirable for a deaf-blind person (if not through the support of an operator). In many cases, the possibility of interaction is dictated by the close relationship that binds the two individuals, such as between mother and son, close relatives or people in close contact. Consequently, the reference scenario makes the solution even more complex. The context, in fact, implicitly assumes social interaction between people who do not have a close relationship.

\section{ARCHITECTURE SUPPORTING MANY-TO-MANY COMMUNICATION}

This section describes how the system architecture has been improved with the aim of supporting a many-to-many communication. In the architecture shown in figure-3, Raspberry-pi based communication system for the deaf and dump people is used. Flex sensors provide signal to the Raspberry-pi and it is converted into communication character. Bluetooth is used to transmit value to mobile device. In particular, it is decided to achieve the many-to-many architecture of the system (fully working from the design point of view) through the use of a tuple_center in order to improve coordination between nodes, making communication easier. The advantages arising from the use of such an abstract computation environment are different. The most important and interesting ones from our point of view are:

- Time uncoupling: it supports communication between time-disjoint processes;

- Primitives: it provides three basic operations: - out: produces a tuple, writing it into tuple_space; 
- in: atomically reads and removes (consumes) a tuple from tuple_space;

- rd: non-destructively reads a tuple form tuple_space.

So we reconsidered the whole architecture, adding to each client a tuple center and leaving the server part unchanged. The new architecture is visible in Figure 3.

Like in previous section, we now examine this new architecture considering structure, behaviour and interaction.

\section{A. Structure}

The new component of the architecture is tuple space. Every client has its own one that will serve as coordination and communication media. The Raspberry $\mathrm{Pi}$ will act as a bridge between the tuple space and the user that are wearing the glove.

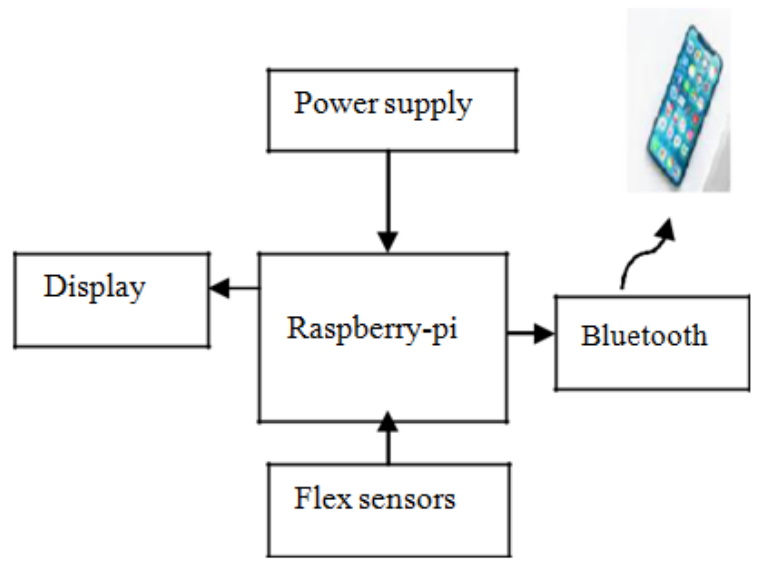

Fig. 3. Architecture of the proposed system

\section{B. Behaviour}

The behaviour of the server remains unchanged and will be modelled through "client registration" and "getting the client list". Instead, the client will not have "peer-to-peer communication" because the communication with the others peer will be delegated to the tuple space. Finally, the behaviour of the tuple_center will be modelled with the three operations described before: out, in and rd.

\section{Interaction}

Peer-topper interaction remains unchanged while peer-topper interaction disappears and it is replaced by tuple space interaction.

\section{CONCLUSION AND FUTURE WORK}

Glove sensor used in this paper is a wearable device that allows one-to-one and one-to-many communication between deaf and blind people and people with no disabilities. An Android app translates the message typed by a deaf-blind person, wearing the glove, using the Malossi alphabet into text. In this paper an extension of GlovePi's current architecture is proposed in order to enable many-to-many communication. The experimental set up of the Glove sensor is shown in Figure 4. We presented a scenario, a conference for deaf and blind people, in which a many-to-many communication is required and then we discussed a possible architecture, exploiting the potentiality of tuple centre as communication and coordination media. As future work, it will be interesting to equip the glove with output sensors (e.g. electromagnetic micro-pulse) can able to reproduce the text written in the Android app, simulating

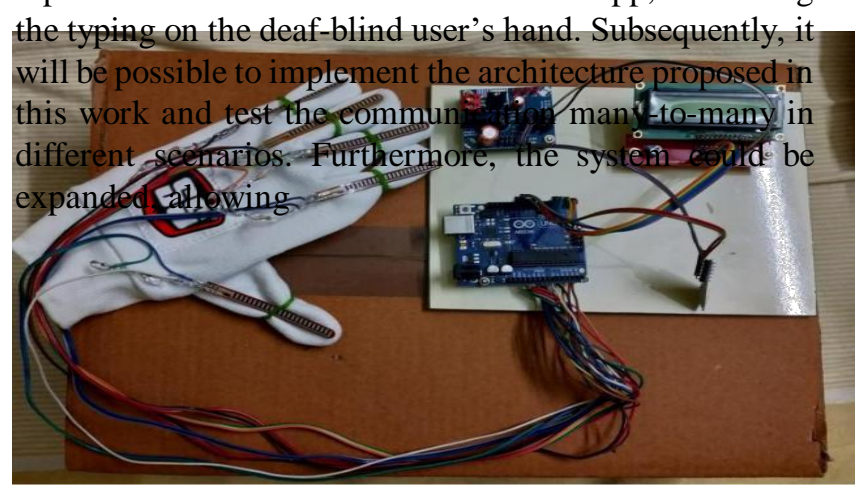

Fig. 4. Glove Sensor's experimental set-up

the mapping of different phonemes to the glove's input/output sensors in order to make possible the communication between people coming from different geographical areas.

\section{REFERENCES}

[1] F. Ramirez-Garibay, C. M. Olivarria, A. F. E. Aguilera, and J. C. Huegel, "Myvox-device for the communication between people: blind, deaf, deaf-blind and unimpaired," in Global Humanitarian Technology Conference (GHTC), 2014 IEEE, pp. 506-509, IEEE, 2014.

[2] M. Gerla, J.-T. Weng, and G. Pau, "Pics-on-wheels: Photo surveillance in the vehicular cloud," in Computing, Networking and Communications (ICNC), 2013 International Conference on, pp. 1123-1127, IEEE, 2013.

[3] M. Federico and M. Furini, "Enhancing learning accessibility through fully automatic captioning," in Proceedings of the International Cross Disciplinary Conference on Web Accessibility, p. 40, ACM, 2012.

[4] M. Roccetti, S. Ferretti, C. E. Palazzi, P. Salomoni, and M. Furini, "Riding the web evolution: from

egoism to altruism," in Consumer Communications and Networking Conference 2008. CCNC 2008. 5th IEEE, pp. 1123-1127, IEEE, 2008.

[5] N. Caporusso, "A wearable Malossi alphabet interface for deafblind people," in Proceedings of the working conference on Advanced visual interfaces - AVI '08, p. 445, 2008.

[6] P. Salomoni, S. Mirri, S. Ferretti, and M. Roccetti,

"Profiling learners with special needs for custom e-learning experiences, a closed case?," in

Proceedings of the 2007 international cross-disciplinary conference on Web accessibility (W4A), pp. 84-92, ACM, 2007.

[7] P. Salomoni, S. Mirri, S. Ferretti, and M. Roccetti,

"A multimedia broker to support accessible and mobile learning through learning objects adaptation," ACM Transactions on Internet Technology (TOIT), vol. 8, no. 2, p. 4, 2008.

[8] R. Jakobson, "Linguistics and communication theory," in Proceedings of symposia in applied mathematics, vol. 12, pp. 245-252, 1961.

[9] S. Mirri, C. Prandi, P. Salomoni, and L. Monti,

"Social location awareness: A prototype of altruistic iot," in New Technologies, Mobility and Security (NTMS), 2016 8th IFIP International Conference on, pp. 1- 5, IEEE, 2016.

[10] S. Mirri, C. Prandi, and P. Salomoni,

"Personalizing pedestrian accessible way-finding with mpass," in Consumer Communications \& Networking Conference (CCNC), 2016 13th IEEE Annual, pp. 11191124, IEEE. 
[11] S. Mirri, C. Prandi, P. Salomoni, F. Callegati, A.

Melis, and M. Prandini, "A service-oriented approach to crowd sensing for accessible smart mobility scenarios," Mobile Information Systems, vol. 2016, 2016.

[12] R.Jakobson, "Linguistics and communication theory," in Proceedings of symposia in applied mathematics, vol. 12, pp. 245-252, 1961

[13] S. Mirri, C. Prandi, P. Salomoni, and L. Monti,

"Fitting like a GlovePi: A wearable device for deaf-blind people," in the proceedings of Consumer Communications \& Networking Conference (CCNC), pp. 1057-1062, IEEE, 2017

[14] R. Sarkar, S. Das, and D. Rudrapal, "A low cost microelectro_mechanical Braille for blind people to communicate with blind or deaf blind people through SMS subsystem," in the proceedings of the 3rd IEEE International Advance Computing Conference, IACC, pp. 1529-1532, 2013.

[15] F. Ramirez-Garibay, C. M. Olivarria, A. F. E.

Aguilera, and J. C. Huegel, "MyVox - Device for the communication between people: Blind, deaf, deaf-blind and unimpaired," in the proceedings of the 4th IEEE Global Humanitarian Technology Conference, GHTC , pp. 506-509, 2014.

[16] TChoudhary, S. Kulkarni, and P. Reddy, "A Braille-based mobile communication and translation glove for deaf-blind people," in the proceedings of International Conference on Pervasive Computing: Advance Communication Technology and Application for Society, ICPC, 2015.

[17] B.D. Gilden and D. L. Jaffe, "Dexter, a robotic hand communication aid for the deaf-blind," International Journal of Rehabilitation Research, vol. 11, no. 2, 1988

[18] H. Brashear, T. Starner, P. Lukowicz, and H.Junker, "Using multiple sensors for mobile sign language recognition," in proceedings of Seventh IEEE International Symposium on Wearable Computers, 2003.

[19] B.U. Gollner, T. Bieling, and G. Joost, "Mobile lorm glove: introducing a communication device for deaf-blind people," in Proceedings of the sixth international conference on tangible, embedded and embodied interaction, pp. 127-130, ACM, 2012.

\section{AUTHORS PROFILE}

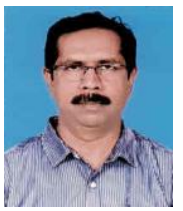

Dr. T. Suresh, M.E., Ph.D, is Professor \& Head, Department of Electronics and Communication Engineering, R.M.K. Engineering college. He obtained his B.E degree in ECE from Madras University, Chennai, M.E., in Microwave and Optical Engineering from Madurai Kamaraj University. He has been in the teaching profession for the past 25 years. He has 2 years of industrial experience. His areas of interest include, Image Processing, VLSI Design and Embedded system. He is a Life member of ISTE and member of IEEE. He has published more than 30 papers in International journals and conferences.

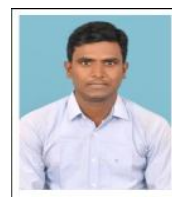

P. Gunasekhar, M.Tech.,(Ph.D), is Assistant Professor, Department of Electronics and Communication Engineering, R.M.K. Engineering college. He obtained his B.E degree in ECE from Anna University, Chennai, M.Tech in Embedded Systems from Jawaharlal Nehru Technological University, Anantapur . He has been in the teaching profession for the past 9.5 years. His areas of interest include, Image Processing, Embedded System and Control systems. He is a Life member of ISTE; he has published papers in International journals and conferences.

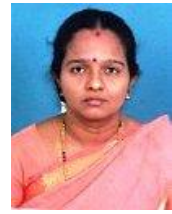

Dr. Z. Brijeth, M.E., Ph.D, is Assistant Professor, Department of Electronics and Instrumentation Engineering, Velammal Engineering college. She obtained her B.E degree in EEE from Manonmaniam Sundaranar University, M.E., in Process Control and Instrumentation from Annamalai University . She has been in the teaching profession for the past 17 years. Her areas of interest include, Controller Design, Computer process control, Microcontroller Design. She is a Life member of ISTE; She has published 10 papers in International journals and conferences.

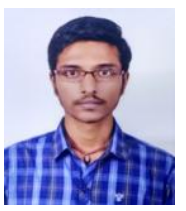

Jasti Tejas, B.E Completed his degree in ECE in R.M.K Engineering College. At present he is Telecom Developer at Cognizant Technology Solutions. He has been in the telecom field for $3+$ years. His areas of interest include Telecommunications, Networking, Mathematics and Science. He is a Life member of ISTE and he aspires to achieve excellence in telecom industry. 